\title{
ON A CERTAIN SET OF LINEAR INEQUALITIES
}

\section{J.G. Kalbfleisch and R.G. Stanton}

(received April 20, 1967)

1. Introduction. In this paper we shall discuss the following set of $\mathrm{n}+1$ linear inequalities:

$$
\begin{aligned}
\mathrm{y}_{0}+\mathrm{y}_{1} & \geq\left(\begin{array}{c}
\mathrm{n} \\
0
\end{array}\right) \\
\mathrm{ny}_{0}+\mathrm{y}_{1}+2 \mathrm{y}_{2} & \geq\left(\begin{array}{l}
\mathrm{n}_{1} \\
\mathrm{n}_{2}
\end{array}\right. \\
(\mathrm{n}-1) \mathrm{y}_{1}+\mathrm{y}_{2}+3 \mathrm{y}_{3} & \geq\left(\begin{array}{l}
\mathrm{n} \\
2
\end{array}\right)
\end{aligned}
$$

(1)

$$
\begin{aligned}
2 y_{n-2}+y_{n-1}+n y_{n} & \geq\left(\begin{array}{c}
n \\
n-1
\end{array}\right) \\
y_{n-1}+y_{n} & \geq\left(\begin{array}{l}
n \\
n
\end{array}\right)
\end{aligned}
$$

If we let $Y=\left(y_{i}\right), C_{n}=\left(\left(\left(_{i}^{n}\right)\right)\right.$, and $Z=\left(z_{i}\right)(i=0,1, \ldots, n)$ be $(n+1)$-dimensional column vectors, and define the $n+1$ by $n+1$ tridiagonal matrix $D_{n}(\phi)$ by

$$
\mathrm{D}_{\mathrm{n}}(\phi)=\left[\begin{array}{ccccccccc}
\phi & 1 & 0 & 0 & 0 & \ldots & 0 & 0 & 0 \\
\mathrm{n} & \phi & 2 & 0 & 0 & \ldots & 0 & 0 & 0 \\
0 & \mathrm{n}-1 & \phi & 3 & 0 & \ldots & 0 & 0 & 0 \\
0 & 0 & \mathrm{n}-2 & \phi & 4 & \ldots & 0 & 0 & 0 \\
\ldots & \ldots & \ldots & \ldots & \ldots & \ldots & \ldots & \ldots \\
0 & 0 & 0 & 0 & 0 & \ldots & \phi & \mathrm{n}-1 & 0 \\
0 & 0 & 0 & 0 & 0 & \ldots & 2 & \phi & \mathrm{n} \\
0 & 0 & 0 & 0 & 0 & \ldots & 0 & 1 & \phi
\end{array}\right],
$$

the set of inequalities (1) may be written

$$
A_{n} Y=C_{n}+Z
$$

where $A_{n}=D_{n}(1)$ and $z_{i} \geq 0(i=0,1, \ldots, n)$. In sections 2 and 3 , we

Canad. Math. Bull. vol. 11, no. 5, 1968. 
consider real solutions of (2), and give expressions for the solution $\mathrm{Y}$ corresponding to a specified vector $Z$ of slack variables. The inequalities (1) arise in connection with a current investigation of some covering properties of groups [4], where it is necessary to find all solutions of (1) in non-negative integers $y_{i}$ with $\Sigma y_{i}$ specified. In section 4, we give an efficient algorithm for obtaining such solutions.

\section{Some properties of the matrix $A_{n}$.}

We begin by considering the set of $n+1$ linear equations

$$
D_{n}(\phi) X=0
$$

where $\mathrm{X}=\left(\mathrm{x}_{\mathrm{i}}\right)(\mathrm{i}=0,1, \ldots, \mathrm{n})$ is a column vector. (3) may be written

$$
(n+1-i) x_{i-1}+\phi x_{i}+(i+1) x_{i+1}=0, \quad-\infty<i<\infty,
$$

with boundary conditions

$$
x_{i}=0 \text { if } i<0 \text { or } i>n \text {. }
$$

Multiplying (4) by $t^{i}$ and summing over all $i$ gives

$$
\text { nt } G(t)-t^{2} G^{\prime}(t)+\phi G(t)+G^{\prime}(t)=0
$$

where $G(t)=\sum x_{i} t^{i}$. The solution of $(6)$ with $G(0)=x_{0}=1$ is

(7) $G(t)=(1+t)^{\frac{n-\phi}{2}}(1-t)^{\frac{n+\phi}{2}}=\sum_{i=0}^{\infty} t^{i} \sum_{r=0}^{i}(-1)^{r}(\underset{r}{(n+\phi) / 2})\left(\begin{array}{c}(n-\phi) / 2 \\ i-r\end{array}\right)$.

It follows that

$$
\left.x_{i}=\sum_{r=0}^{i}(-1)^{r} \underset{r}{(n+\phi) / 2}\right)\left(\begin{array}{c}
(n-\phi) / 2 \\
i-r
\end{array}\right) .
$$

The boundary conditions (5) are satisfied if and only if $n+\phi$ is an even integer and $-\mathrm{n} \leq \phi \leq \mathrm{n}$; thus (3) has a nontrivial solution $\mathrm{X}$ if and only if

$$
\phi=\phi_{j}=n-2 j \quad(j=0,1, \ldots, n) .
$$


The solution vector $x_{j}=\left(x_{i j}\right)$ corresponding to $\phi_{j}$ is given by

$$
x_{i j}=\sum_{r=0}^{i}(-1)^{r}\left(\begin{array}{c}
n-j \\
r
\end{array}\right)\left(\begin{array}{c}
j \\
i-r
\end{array}\right)
$$

We may now determine the eigenvalues and eigenvectors of $A_{n}$; for the equations $A_{n} X=\lambda X$ may be written $D_{n}(\phi) X=0$ with $\phi=1-\lambda$. Thus $\mathrm{A}_{\mathrm{n}}$ has eigenvalues

$$
\lambda_{j}=1-\phi_{j}=1-n+2 j \quad(j=0,1, \ldots, n)
$$

and the eigenvector corresponding to $\lambda_{j}$ is $x_{j}=\left(x_{i j}\right)$. It also follows that $\operatorname{det} A_{n}=\pi \lambda_{j}$, which is zero for $n$ odd, and equal to $(-1)^{n / 2}(n+1)(n-1)^{2}(n-3)^{2} \ldots 3^{2} \cdot 1^{2}$ for $n$ even. Det $A_{n}$ may also be obtained simply by direct expansion and recursion. In fact, the determinant of $D_{n}(\phi)$ was evaluated by J. J. Sylvester [5] as early as 1854 .

Let $\mathrm{x}=\left(\mathrm{x}_{0}\left|\mathrm{x}_{1}\right| \ldots \mid \mathrm{x}_{\mathrm{n}}\right)$ be the $\mathrm{n}+1$ by $\mathrm{n}+1$ modal matrix whose columns are the eigenvectors $x_{0}, x_{1}, \ldots, x_{n}$. The matrix $x$ was computed for small values of $n$, and it was noted that each row of $X$ is orthogonal to all but one of the columns of $X$. Consequently the following lemma was obtained.

LEMMA. Let $S_{i j}=\sum_{\alpha=0}^{n} x_{i \alpha} x_{\alpha j}$, where $x_{i j}$ is defined by (10). Then

$$
S_{i j}=0 \text { if } j \neq n-i ; S_{i, n-i}=(-1)^{i} 2^{n}
$$

Proof: Put $H_{j}(u)=\sum_{i} S_{i j} u^{i}$. Then, using the generating function

$$
G_{j}(t)=\sum_{i} x_{i j} t^{i}=(1+t)^{j}(1-t)^{n-j}
$$

corresponding to $\phi_{j}$ we get 


$$
\begin{aligned}
H_{j}(u) & =\underset{i k}{\sum} x_{i k} x_{k j} u^{i}=\sum_{k} x_{k j}(1+u)^{j}(1-u)^{n-j} \\
& =(1-u)^{n}\left(1+\frac{1+u}{1-u}\right)^{j}\left(1-\frac{1+u}{1-u}\right)^{n-j}=2^{n}(-u)^{n-j} .
\end{aligned}
$$

By comparing coefficients of powers of $u$ we obtain (12).

Thus the $i^{\text {th }}$ row of $\mathrm{X}$ is orthogonal to every column of $\mathrm{X}$ except the $(n-1)^{\text {th }}(i=0,1, \ldots, n)$ and

$$
x^{-1}=2^{-n}\left(x_{n}\left|-x_{n-1}\right| x_{n-2}\left|-x_{n-3}\right| \cdots \mid(-1)^{n} x_{0}\right)
$$

Let $\Lambda$ be the diagonal matrix with diagonal entries $\lambda_{0}, \lambda_{1}, \ldots, \lambda_{n}$, so that $\mathrm{A}_{\mathrm{n}} \mathrm{X}=\mathrm{X} \wedge$. Then if $\mathrm{n}$ is even,

$$
A_{n}^{-1}=x \wedge^{-1} x^{-1}
$$

We are indebted to Professor D. A. Sprott for pointing out a connection with probability theory. If $n$ is even, $\frac{1}{n} D_{n}(0)$ is the matrix of transition probabilities for the Ehrenfest Model. A discussion of some of the properties of this matrix appears in [1] and [3]. The arguments given here are somewhat simpler than theirs because the Lemma makes the derivation of $\mathrm{X}^{-1}$ almost trivial.

3. Real Solutions of $\mathrm{A}_{\mathrm{n}} \mathrm{Y}=\mathrm{C}_{\mathrm{n}}+\mathrm{Z}$. If $\mathrm{n}$ is even, $\mathrm{A}_{\mathrm{n}}$ is nonsingular, and there will be a unique solution $Y$ of (2) corresponding to each vector $Z$. Since $C_{n}=X_{n}$, the eigenvector corresponding to $\lambda_{\mathrm{n}}=\mathrm{n}+1$, we have

$$
Y=A_{n}^{-1}\left(C_{n}+Z\right)=\frac{1}{n+1} C_{n}+A_{n}^{-1} Z
$$

If the slack variables $z_{i}$ are all non-negative, $Y$ is a solution of (1).

If $\mathrm{n}$ is odd, say $\mathrm{n}=2 \mathrm{k}+1, \mathrm{~A}_{\mathrm{n}}$ is singular and the situation is slightly more complicated. Since $\mathrm{x}_{0}, \mathrm{x}_{1}, \ldots, \mathrm{x}_{\mathrm{n}}$, are linearly independent, everyvector $\mathrm{Y}$ may be expressed as a linear combination 
of them. If $\mathrm{Y}=\Sigma \mathrm{a}_{\mathrm{j}} \mathrm{X}_{\mathrm{j}}$ is a solution,

$$
A_{n} Y=\Sigma a_{j} A_{n} X_{j}=\Sigma a_{j} \lambda_{j} X_{j}=C_{n}+Z
$$

Since $C_{n}=X_{n}$ and $\lambda_{k}=0, Z$ must lie in the subspace spanned by $\mathrm{x}_{0}, \mathrm{x}_{1}, \ldots, \mathrm{x}_{\mathrm{k}-1}, \mathrm{x}_{\mathrm{k}+1}, \ldots, \mathrm{x}_{\mathrm{n}}$. But, by the Lemma,

$$
\sum_{\alpha} x_{k+1, \alpha} x_{\alpha i}=0, \quad i \neq k
$$

and therefore the slack variables $\mathrm{z}_{\alpha}$ must satisfy

$$
\sum_{\alpha} \mathrm{z}_{\alpha} \mathrm{x}_{\mathrm{k}+1, \alpha}=0
$$

If $\mathrm{Z}$ is any vector satisfying (16), then $\mathrm{Z}=\Sigma \mathrm{b}_{j} \mathrm{X}_{j}$ with $\mathrm{b}_{k}=0$, and corresponding to $\mathrm{Z}$ are solutions

$$
Y=\frac{1}{n+1} C_{n}+\sum_{j \neq k} \frac{b_{j}}{\lambda_{j}} X_{j}+t X_{k}
$$

where $t$ is an arbitrary real number. Let $\hat{\Lambda} *$ be the diagonal matrix with diagonal entries $\lambda_{0}, \lambda_{1}, \ldots, \lambda_{k-1}, 1, \lambda_{k+1}, \ldots, \lambda_{n}$, and let $B$ be the column vector $\left(b_{j}\right)$. Then $z=X B$, and thus $B=X^{-1} Z$. We may now rewrite (17) in the form

$$
Y=\frac{1}{n+1} C_{n}+X \wedge * *^{-1} X^{-1} Z+t X_{k}
$$

If all the slack variables $\mathrm{z}_{\alpha}$ are non-negative, $\mathrm{Y}$ is a solution of (1).

A convenient algorithm for numerical computation of real solutions $\mathrm{Y}$ of the equations $\mathrm{AY}=\mathrm{B}$, where $\mathrm{Y}$ and $\mathrm{B}$ are column vectors and $A$ is an arbitrary nonsingular tridiagonal matrix, is described by Henrici [2, page 350]. This algorithm depends upon a rather interesting factorization of a tridiagonal matrix into two "bidiagonal" matrices.

4. Solutions of (1) in non-negative integers. The inequalities (1) arise in connection with a covering problem [4] where $\mathrm{y}_{\alpha}$ represents the number of elements of a certain type in a covering set. Consequently 
the $\mathrm{y}_{\alpha}{ }^{\prime} \mathrm{s}$ and $\mathrm{z}_{\alpha}{ }^{\prime} \mathrm{s}$ must be non-negative integers. The problem is to construct a covering set with as few members as possible - that is, with $\Sigma_{y_{\alpha}}$ as small as possible. Adding the equations (2) gives

$$
(\mathrm{n}+1) \Sigma_{\mathrm{y}_{\alpha}}=2^{\mathrm{n}}+\Sigma_{\mathrm{z}}
$$

so that $\Sigma y_{\alpha} \geq 2^{n} /(n+1)$. This is not, however, a sufficient condition for the existence of a solution of (1). Furthermore not every solution $\mathrm{Y}$ corresponds to a covering set. Thus it is often necessary to consider several totals $\Sigma_{y_{\alpha}}$, beginning with the least integer greater than or equal to $2^{n} /(n+1)$. It is, however, necessary to consider only solutions with $\mathrm{y}_{0}=1$ since any covering set will be isomorphic to one with $\mathrm{y}_{0}=1$.

In this section we give an efficient algorithm for finding all solutions of (1) with $y_{0}=1$ and $\Sigma y_{\alpha} \leq m$. The latter condition is equivalent to insisting that the total slack $\Sigma_{z_{\alpha}}$ be at most $T=2^{n}-m(n+1)$. The algorithm is easily programmed for a computer, and may be generalized to yield the non-negative integer solutions of many sets of linear inequalities whose matrices of coefficients are tridiagonal.

The algorithm is represented pictorially by the directed graph in Figure 1. Vertices represent operations, and edges indicate the order in which they are performed. First, we give the operations corresponding to the vertices and the rules for moving from one to another. Then we shall explain the algorithm and give an example.

$S(\operatorname{star} t):$ Put $y_{-1}=0, y_{0}=1, z_{0}=T, k=0$. Go to $A$.

A: Calculate $R_{k}=\left(\begin{array}{l}n \\ k\end{array}\right)+z_{k}-y_{k}-(n-k+1) y_{k-1}$. If $R_{k}<0$, go to C; if $R_{k} \geq 0$, go to $B$.

B: Calculate $y_{k+1}=\left[R_{k} /(k+1)\right], z_{k}=(k+1) y_{k+1}+y_{k}+(n-k+1) y_{k-1}-\left(\begin{array}{l}n \\ k\end{array}\right)$

$$
\begin{aligned}
& \text { and } z_{k+1}=T-z_{1}-z_{2}-\ldots-z_{k} \text {. If } z_{k}<0 \text {, go to } C \text {; if } z_{k} \geq 0 \text { and } \\
& k<n-1 \text {, go to } G \text {; if } z_{k} \geq 0 \text { and } k=n-1 \text {, go to } F \text {. }
\end{aligned}
$$

C: Select the largest $j<k$ for which $z_{j} \neq 0$ and go to D. If $z_{j}=0$ for all $j<k$, go to $E$. 
D: Decrease $z_{j}$ by 1 and put $z_{j+1}=T-z_{0}-z_{1} \ldots-z_{j}$. Put

$$
\mathrm{k}=\mathrm{j} \text { and go to } A \text {. }
$$

E: Algorithm terminates.

F: Put $z_{n}=y_{n-1}+y_{n}-1 . Y$ is a solution with slack $Z$. Go to $C$.

$G$ : Increase $k$ by 1 and go to $A$.

The algorithm examines all possible slack vectors $Z$ with $\Sigma_{\alpha} \leq \mathrm{T}$. We begin with $\mathrm{Z}=(\mathrm{T}, 0,0, \ldots, 0)$, and change $\mathrm{Z}$ in such a way that

$$
\|z\|=z_{0}(T+1)^{n}+z_{1}(T+1)^{n-1}+\ldots+z_{n-1}(T+1)+z_{n}
$$

is steadily decreasing. Suppose that after a number of steps we reach A with $z=\left(z_{0}, z_{1}, \ldots, z_{k}, 0, \ldots, 0\right)$, and have solved the first $k$ equations of (2) for $y_{0}, y_{1}, \ldots, y_{k}$. The $k^{\text {th }}$ equation (numbering from 0 ) is

$$
(k+1) y_{k+1}+y_{k}+(n-k+1) y_{k-1}=\left(\begin{array}{l}
n \\
k
\end{array}\right)+z_{k} \text {, }
$$

and we wish to solve this for $y_{k+1}$. Since $z_{k}$ contains all the available slack, $(k+1) y_{k+1}$ cannot be larger than $R_{k}$. Thus if $R_{k}$ is negative, we cannot solve for $\mathrm{y}_{\mathrm{k}+1}$, and must alter the values of $z_{k-1}, z_{k-2}, \ldots$ (step C). If $R_{k} \geq 0$, we must choose $y_{k+1}$ so that $\|z\|$ will be as large as possible (step B). This means that we want as much slack as possible in the $k^{\text {th }}$ equation, and so $y_{k+1}$ must be chosen as large as possible. We must now recalculate $z_{k}$, which becomes the actual slack in the $k^{\text {th }}$ equation, and place all remaining slack at $z_{k+1}$.

If the new value of $z_{k}$ is negative, we have reached a contradiction and must go to $\mathrm{C}$ where the values of $z_{k-1}, z_{k-2}, \ldots$ will be altered. If $z_{k} \geq 0$ and $k=n-1$, we have a solution $Y$ (step F). The actual slack in the $n^{\text {th }}$ equation will be $y_{n-1}+y_{n}-1$, and the solution has total slack $T-z_{n}+y_{n-1}+y_{n}-1$. To obtain other solutions we go to $C$ where $z_{n-1}, z_{n-2}, \ldots$ are changed. Finally, if $z_{k} \geq 0$ and $k<n-1$, we replace $k$ by $k+1$ (step G), and then return to $A$ to solve the next equation for the next $Y$ value. 
When we arrive at $C$ from $A, B$, or $F$, we must alter the first $k$ slack variables $z_{0}, z_{1}, \ldots, z_{k-1}$ in such a way that $\|z\|$ decreases, but does so as little as possible. This is accomplished by decreasing by one the last nonzero $z_{j}$. Thus in step $C$ we select the largest $j<k$ with $z_{j} \neq 0$. If no such $j$ exists (as must eventually happen) the algorithm terminates at E. Otherwise (step D) we decrease $z_{j}$ by one, put all remaining slack at $z_{j+1}$, and return to A. Since $z_{0}, z_{1}, \ldots, z_{j-1}$ have not been changed, $y_{0}, y_{1}, \ldots, y_{j}$ also remain fixed, and we begin our calculations with $y_{j+1}$ - that is, with $k=j$ in step $A$.

As an example, we take $\mathrm{n}=5, \Sigma \mathrm{y}_{\alpha} \leq 7$, and total allowable slack $\mathrm{T}=10$. The first column of Table 1 names the step, and the remaining columns give the values of $k, R_{k}, j, Z$, and $Y$ at that step. Opposite a step we have entered only the values which are calculated at that step, and other variable values remain unchanged from the preceding step. The first few iterations are rather uninteresting and no solutions are obtained. We omit them, and begin at $A$ with $z_{0}=2, z_{1}=8, y_{0}=1, y_{1}=2$, and $k=1$. We give several steps during which a solution $Y=(1,2,2,0,1,1)$, $Z=(2,6,0,0,1,1)$ is obtained $(F)$. If the algorithm is continued to completion, a total of seventeen solutions of (1) with $\mathrm{y}_{0}=1$ and $\Sigma \mathrm{y}_{\alpha} \leq 7$ are obtained, two with $\Sigma \mathrm{y}_{\alpha}=6$, and fifteen with $\Sigma \mathrm{y}_{\alpha^{\prime}}=7$. The computations required only a few seconds on the IBM 7040 computer. 


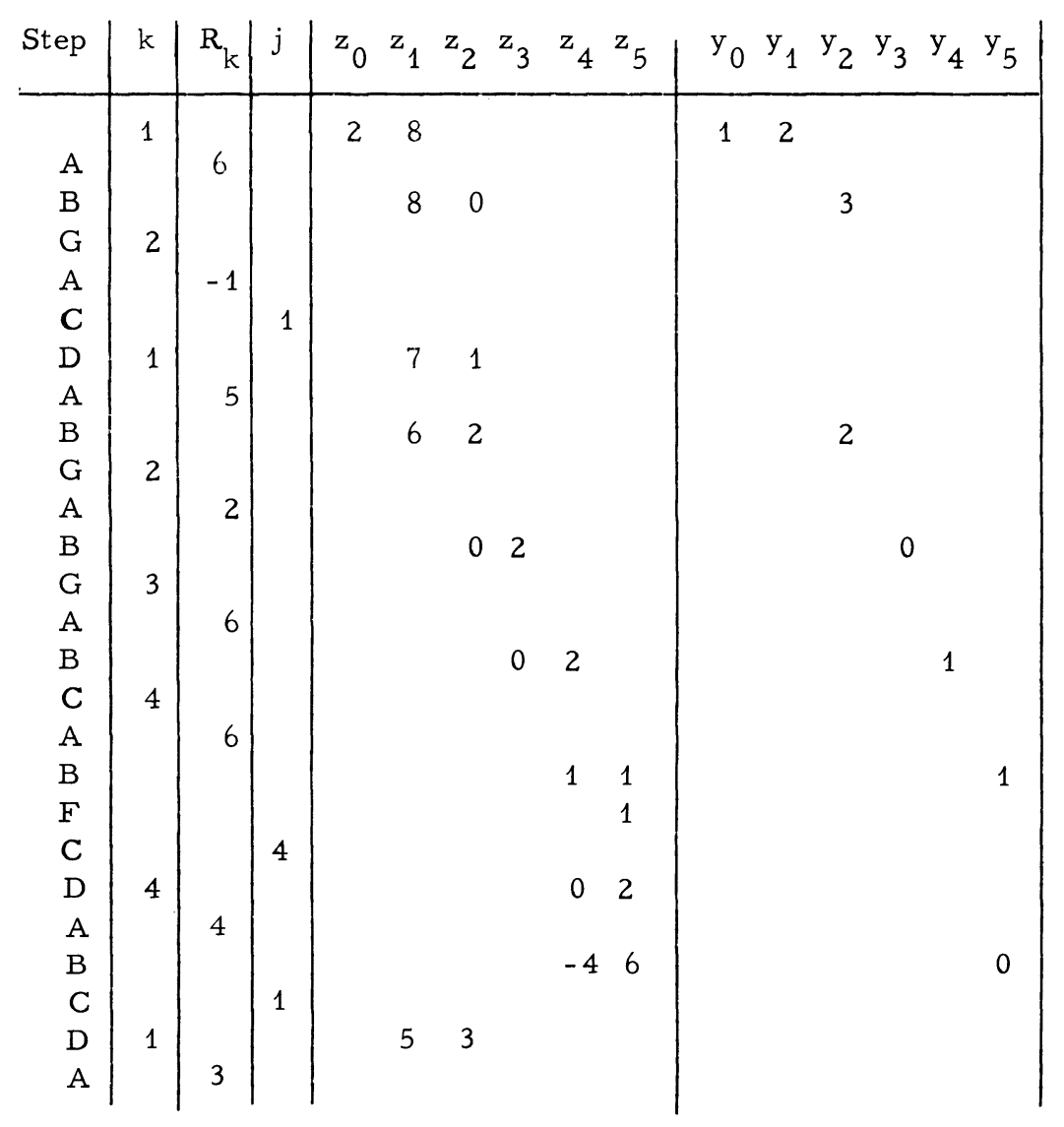

Table 1

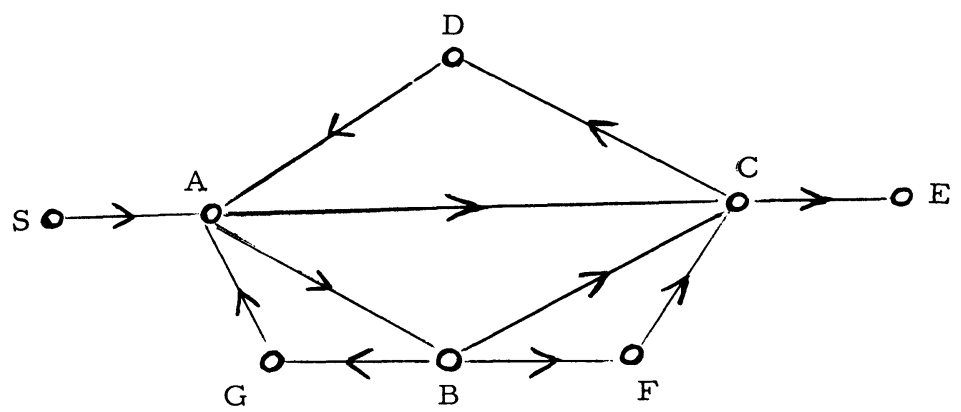

Figure 1

689 


\section{REFERENCES}

1. D.R. Cox and H.D. Miller, The theory of stochastic processes. (Methuen and Co. Ltd., London, 1965) 129-132.

2. P. Henrici, Discrete variable methods in ordinary differential equations. (Wiley, 1962).

3. Mark Kac, Random walk and the theory of Brownian motion, American Mathematical Monthly 54 (1947), 369-391.

4. R.G. Stanton and J.G. Kalbfleisch, Covering problems for dichotomized matchings. Aequationes Mathematicae 1 (1968) 94-103.

5. J.J. Sylvester, Théorème sur les déterminants de M. Sylvester, Nouv. Annales de Math. 13 (1854) 305.

York University

Toronto 